\title{
Deregulated expression of pro-survival and pro-apoptotic p53-dependent genes upon Elongator deficiency in colon \\ cancer cells
}

\author{
Isabelle Cornez $^{a, b}$, Catherine Creppe ${ }^{a, b}$, Magali Gillard ${ }^{a, b}$, Benoît Hennuy ${ }^{d}$, \\ Jean-Paul Chapelle ${ }^{a, b}$, Emmanuel Dejardin ${ }^{a, c}$, Marie-Paule Merville ${ }^{a, b}$, \\ Pierre Close ${ }^{a, b, 1}$, Alain Chariot ${ }^{a, b, *}$ \\ a Unit of Signal Transduction, Interdisciplinary Cluster for Applied Genoproteomics, University of Liège, Sart-Tilman, \\ B-4000 Liège, Belgium \\ ${ }^{\mathrm{b}}$ Medical Chemistry Unit, University of Liège, Sart-Tilman, B-4000 Liège, Belgium \\ ${ }^{c}$ Virology/Immunology Unit, University of Liège, Sart-Tilman, B-4000 Liège, Belgium \\ ${ }^{\mathrm{d}}$ GIGA Transcriptomics Facility, University of Liège, Sart-Tilman, B-4000 Liège, Belgium
}

\section{A R T I C L E I N F O}

Article history:

Received 31 January 2008

Accepted 7 March 2008

\section{Keywords:}

IKK-associated protein (IKAP/hELP1)

Elongator

p53

Familial dysautonomia

Serum- and glucocorticoid-induced protein kinase (SGK)

BCL2-associated X protein (BAX)

\begin{abstract}
A B S T R A C T
Elongator, a multi-subunit complex assembled by the IkappaB kinase-associated protein (IKAP)/hELP1 scaffold protein is involved in transcriptional elongation in the nucleus as well as in tRNA modifications in the cytoplasm. However, the biological processes regulated by Elongator in human cells only start to be elucidated. Here we demonstrate that IKAP/hELP1 depleted colon cancer-derived cells show enhanced basal expression of some but not all pro-apoptotic p53-dependent genes such as BAX. Moreover, Elongator deficiency causes increased basal and daunomycin-induced expression of the pro-survival serum- and glucocorticoid-induced protein kinase (SGK) gene through a p53-dependent pathway. Thus, our data collectively demonstrate that Elongator deficiency triggers the activation of p53dependent genes harbouring opposite functions with respect to apoptosis.
\end{abstract}

(C) 2008 Elsevier Inc. All rights reserved.

\footnotetext{
* Corresponding author at: Laboratory of Medical Chemistry/GIGA-R, Tour GIGA +2 B34, CHU Sart-Tilman, 4000 Liège, Belgium.

Tel.: +32 436624 72; fax: +32 43664534 .

E-mail address: Alain.chariot@ulg.ac.be (A. Chariot).

${ }^{1}$ Present address: Cancer Research UK London Research Institute, Clare Hall Laboratories, South Mimms, United Kingdom.

Abbreviations: B2M, beta-2-microglobulin; BARD1, BRCA1-associated RING domain 1; BAX, BCL2-associated X protein; BTG2, B-cell translocation gene 2; CDK9, cyclin-dependent kinase 9; DMEM, Dulbecco's modified Eagle's medium; DUSP4, dual-specificity phosphatase 4; Elp, Elongator protein; EMEM, Eagle's modified essential medium; FACT, facilitates chromatin transcription; GFP, green fluorescent protein; HA, haemagglutinin; hELP1, human ELP1; IKAP, IKK-associated protein; IKK, inhibitor of kappa light chain gene enhancer in B cells kinase complex; NR2F2, nuclear receptor subfamily 2, group F, member 2; p21, cyclin-dependent kinase inhibitor 1A; PARP, poly (ADP-ribose) polymerase 1; PKIB, protein kinase, cAMP-dependent catalytic, inhibitor beta; RhoE/Rnd3, Rho family GTPase 3; SESN2, sestrin 2; SGK, serum- and glucocorticoid-induced protein kinase; shRNA, short hairpin RNA; TFIIF, general transcription factor IIF; TFIIH, general transcription factor IIH; TNFRSF10D, tumor necrosis factor receptor superfamily, member 10D.
} 0006-2952/\$ - see front matter (C) 2008 Elsevier Inc. All rights reserved.

doi:10.1016/j.bcp.2008.03.006 


\section{Introduction}

Elongator, a highly conserved complex of 6 subunits (Elp1Elp6), was initially identified as a complex associated with hyperphosphorylated form of yeast RNA polymerase II and subsequently isolated from human cells as well [1-3]. Elongator requires the scaffold IKK-associated protein (IKAP)/ELP1/hELP1 for assembling into a functional complex [4]. Moreover, Elp3, the catalytic subunit, harbours motifs found in the GNAT family of histone acetyltransferases (HATs) [5] and is essential for the ability of Elongator to target histone $\mathrm{H} 3$ in vitro $[2,3,6]$ and in vivo [7]. These observations combined with reports showing an association of Elongator with several nascent RNAs in yeast [8] and with the preferential recruitment of Elongator to the transcribed regions of human genes [9-11], strongly support a role for Elongator in transcriptional elongation. Meanwhile, other reports also provided experimental evidence for a role of Elongator in exocytosis and tRNA modification [12-14]. Still and although these reports brought significant insights regarding the biological roles of Elongator, it is currently unclear whether this complex harbours multiple functions in distinct cell compartments or whether a single function indirectly regulates the others [15].

Loss of function models in distinct organisms helped to decipher the Elongator-dependent pathways and their biological relevance. Elongator yeast mutants (elp phenotypes) have slower growth adaptation and temperature sensitivity at $39{ }^{\circ} \mathrm{C}$ [5]. The Elongata mutants of Arabidopsis thaliana, which have point mutations in genes coding for the yeast Elongator subunit Elp1, Elp3 and Elp4, are characterized by reduced root growth due to decreased cell division rate, which supports a role for Elongator in cell proliferation in plants [16].

Consequences of impaired Elongator function in humans are exemplified by familial dysautonomia (FD), an autosomal recessive disease, ranging among the most frequent hereditary sensory and autonomic neuropathies and characterized by defects in the development and maintenance of neurons of the autonomic and sensory systems $[17,18]$. FD is due to a mutation in a splice site of the IKBKAP gene, which ultimately causes decreased expression of IKAP/hELP1 in a tissue-specific manner $[19,20]$. Although the molecular and cellular mechanisms underlying this neuro-developmental and neurodegenerative genetic disorder remain largely undefined, impaired cell motility may account for this disease as a variety of human cells depleted for IKAP/hELP1 harboured defects in cell migration [11]. Thus, these studies collectively demonstrate that impaired Elongator function causes multiple cellular defects.

Signalling pathways triggered by DNA-damaging agents or many other forms of cellular stress ultimately regulate gene transcription. Whereas pre-initiation and initiation steps of transcription involve multiple levels of regulation, there are now accumulating evidences for transcriptional elongation being a tightly regulated process that requires a plethora of proteins, for example the elongation factors [21] such as TFII, $\mathrm{TFIIH}$, and the multi-protein complex FACT [22]. In this context, gene transcription in response to DNA-damaging agents relies on gene- and signal-specific elongation factors [23]. It is currently unknown whether Elongator is required for gene transcription in response to DNA-damaging agents and whether Elongator deficiency impairs the ability of the other elongating factors to properly transcribe their target genes.

In order to further characterize the Elongator-dependent pathways and concomitantly learn more about the cellular response to DNA-damaging agents, we generated colon cancer-derived cells depleted for IKAP/hELP1 and showed here that Elongator is dispensable for both basal- and daunomycin-induced expression of some p53-dependent genes involved in cell cycle arrest such as $p 21$. Interestingly, Elongator deficiency activates selected p53-dependent pathways, as evidenced by enhanced basal expression of BAX. Moreover, IKAP/hELP1 depleted cells show enhanced basal and daunomycin-induced mRNA expression of the serumand glucocorticoid-inducible kinase SGK through a p53dependent pathway. Therefore, our results represent the first experimental evidence for a link between impaired Elongator function and the activation of selected p53-dependent pathways.

\section{Materials and methods}

\subsection{Cell culture and reagents}

HCT116 and p53 deficient HCT116 cells (a gift from Dr. Bert Vogelstein, Ludwig Center for Cancer Genetics and Therapeutics and Howard Hughes Medical Institute at Johns Hopkins Kimmel Cancer Center, Baltimore, MD, USA) were maintained in Mac Coy medium supplemented with $10 \%$ FBS and $1 \%$ antibiotic whereas 293FT and HT29 cells were maintained in DMEM or EMEM supplemented with $10 \%$ fetal bovine serum (Life Technologies), 1\% L-glutamine and 1\% antibiotics, respectively. Human primary fibroblasts, a gift from Dr. Charles Lambert (Laboratory of Connective Tissues Biology, Interdisciplinary Cluster for Applied Genoproteomics, University of Liege, Belgium), were cultured in DMEM supplemented with 10\% FBS, $1 \%$ antibiotics and 1\% L-glutamine. Daunomycin hydrochloride and actinomycin D were purchased from Sigma-Aldrich (Saint Louis, MO, USA). The monoclonal anti-IKAP/hELP1 and -PARP antibodies were purchased from BD Biosciences Pharmingen (San Jose, CA, USA) whereas the anti-Elp3 antibody was previously described [11]. The anti-p21 and -BAX antibodies were from Calbiochem (San Diego, CA, USA) whereas the anti-serine 15 phospho p53 (Pp53), -p53, -FACT, -CDK9 and - $\alpha$-tubulin antibodies were from Santa Cruz Biotechnologies (Santa Cruz, CA, USA). The monoclonal anti-caspase 3, RhoE/Rnd3 and actin antibodies were from Alexis (Lausen, Switzerland), Upstate (Millipore company, Billerica, MA, USA) and Sigma (St. Louis, MO, USA), respectively. Theses antibodies were used in Western blot experiments, according to standard protocols.

\subsection{RNAi transfection and lentiviral cell infection}

RNAi oligos were synthesized by Dharmacon Research (sequences available upon request), and were transfected into human primary fibroblasts using calcium phosphate. Cells were lyzed $48 \mathrm{~h}$ post-transfection, and anti-IKAP/hELP1 and -ELP3 Western blots were subsequently performed. The pLL3.7 shRNA IKAP 1 and GFP lentiviral constructs were 
previously described [11] whereas the shRNA IKAP 2 construct was generated by subcloning another IKAP sequence (available upon request) into the pLL3.7 vector. Lentiviral IKAP/ hELP1 and GFP shRNAs constructs were also generated by subcloning the corresponding sequences into the BLOCK-iT lentiviral RNAi expression system, according to the protocol provided by the manufacturer (Invitrogen, Carlsbad, CA, USA). Infected cells were subsequently selected with blasticidin (Invivogen, San Diego, CA, USA) $(10 \mu \mathrm{g} / \mathrm{ml})$ to produce stably transduced cells. Clones were selected based on residual IKAP/ hELP1 protein levels.

\subsection{Total RNA extraction, micro-array analysis}

Stably transduced IKAP/hELP1 shRNA or GFP shRNA HCT116 cells were left untreated or stimulated with daunomycin for $2 \mathrm{~h}$. Those experiments were repeated with the stably transduced IKAP/hELP1 shRNA or GFP shRNA p53 deficient HCT116 cells in order to reach a total of 8 distinct experimental conditions. Triplicates from each 8 experimental conditions were subjected to total RNA extractions using the RNeasy Minit kit (Qiagen) and the resulting total RNAs from those triplicates were pooled. The integrity of the RNAs was confirmed with the Agilent Bioanalyser using the RNA 6000 Nano kit (Agilent, Santa Clara, CA, USA). Double-stranded cDNAs were generated using the Superscript II RT kit (Invitrogen, Carlsbad, CA, USA). Subsequently, biotin-labelled cRNA was generated using the GeneChip One-Cycle Target Labeling kit (Affymetrix, Santa Clara, CA, USA). cRNAs were hybridized with the Human Genome U133A array, which harbours $>22,000$ probe sets (Affymetrix) targeting. Briefly, double-stranded cDNA was synthesized routinely from $5 \mu \mathrm{g}$ of total RNA primed with a poly-(dT) -T7 oligonucleotide. The cDNA was used in an in vitro transcription (IVT) reaction in the presence of T7 RNA polymerase and biotin-labelled modified nucleotides during $16 \mathrm{~h}$ at $37^{\circ} \mathrm{C}$. Biotinylated cRNA was purified and then fragmented (35-200 nucleotides), together with hybridization controls and hybridized to the microarrays for $16 \mathrm{~h}$ at $45^{\circ} \mathrm{C}$. Using the Fluidics Station (Affymetrix), the hybridized biotin-labelled cRNA was revealed by successive reactions with streptavidin R-phycoerythrin conjugate, biotinylated antistreptavidine antibody and streptavidin Rphycoerythrin conjugate. The arrays were finally scanned in an Affymetrix/Hewlett-Packard GeneChip Scanner 3000. Data were processed using the MAS 5.0 software (Affymetrix).

\subsection{Real-time PCRs}

Quantitative real-time PCR were performed with TaqMan 7000 SDS (Applied Biosystems, Foster City, CA, USA), using SYBR Green detection. Primer sequences for p21 [24], B2M and IKAP/ hELP1 [11] were previously described and are available upon request, as are the primer sequences for SGK, TNFRSF10D, SESN2, BTG2, PKIB and BAX.

\subsection{Chromatin immunoprecipitation (ChIP) assay}

Chromatin immunoprecipitation assays were carried out as previously described [11] with stably transduced IKAP/hELP1 or GFP shRNA HCT116 cells $\left(30 \times 10^{6}\right.$ cells/condition). These were left untreated or treated with actinomycin D (20 nM) for 2 and/or $8 \mathrm{~h}$. Sonicated extract were precleared by $1 \mathrm{~h}$ incubation with protein A-agarose/Herring sperm DNA (Santa Cruz/ Sigma). Immunoprecipitations were performed by incubating the sonicated extracts with $2.5 \mu \mathrm{g}$ of anti-HA (Y-11, negative control), anti-p53 (FL-393) or anti-RNA polymerase II (H-224) (Santa Cruz biotechnologies) overnight at $4{ }^{\circ} \mathrm{C}$. Protein Aagarose was subsequently added and mixed for a further $1 \mathrm{~h}$ at $4{ }^{\circ} \mathrm{C}$. Phenol/chloroform purified DNA fragments were analysed by real-time PCR using the SYBER Green detection. For the normalization of the RNAPII ChIPs, the signal of a noncoding region downstream from the albumin gene [10] was used to compensate for possible fluctuations arising during handling.

\subsection{Caspase $3 / 7$ cleavage and cellular viability}

For the quantification of cellular apoptosis, HCT116 cells infected with the pLL3.7 shRNA GFP, IKAP 1 or IKAP 2 lentiviral constructs were seeded in 96-well plates $\left(1 \times 10^{4}\right.$ cells/well) and left unstimulated or treated with actinomycin D (20 nM). Apoptosis was measured by quantification of both caspase 3 and 7 activities, using the luminometric Caspase-Glo 3/7 assay (Promega, Madison, USA), according to the manufacturer's protocol, and a Victor2 multilabel counter (PerkinElmer, Waltham, Massachusetts, USA). For assessment of cellular viability, HCT116 cells infected with the pLL3.7 shRNA GFP, IKAP 1 or IKAP 2 lentiviral constructs were seeded in 96-well plates $\left(1 \times 10^{4}\right.$ cells/well $)$ and left unstimulated or treated with actinomycin $\mathrm{D}(20 \mathrm{nM})$ and viability was measured using the CellTiter-Glo Luminescent Cell Viability Assay (Promega, Madison, USA), according to the manufacturer's instructions. Statistical analysis of the data was performed by one-way ANOVA with Dunn's multiple comparison test using Prism 4.00 Software (Graph pad, San Diego, USA), with statistical significance accepted at $P<0.05$.

\section{Results \\ 3.1. IKAP/hELP1 depletion modulates gene expression in a cell-type specific manner}

We previously demonstrated that Elongator is required for the expression of a subset of genes in HeLa cells, many of which encoding proteins involved in cell motility. Elongator deficient cells indeed showed defect in cell migration, as judged by multiple assays [11]. To further characterize the Elongatordependent biological processes and because the links between the signalling pathways triggered by DNA-damaging agents and gene expression remain poorly characterized, we hypothesized that IKAP/hELP1 deficient cells may harbour expression defects of a subset of genes upon stimulation with a DNA-damaging agent. To experimentally address this hypothesis, we first generated colon cancer-derived cells deficient for IKAP/hELP1 through RNA interference by infecting HCT116 cells with a lentivirus delivering small hairpin RNAs targeting either the IKAP/hELP1 transcript, or GFP as a negative control ("IKAP/hELP1 shRNA" and "GFP shRNA", respectively). IKAP/hELP1 depletion in those cells did not affect 
their proliferation (data not shown). We subsequently left the resulting cells untreated or stimulated with daunomycin for $2 \mathrm{~h}$. Decreased IKAP/hELP1 expression was indeed observed in IKAP/hELP1 shRNA versus GFP shRNA cells, both at the mRNA (Fig. 1A) and at the protein levels (Fig. 1B, compare lanes 1 and 2, top panel). Moreover, decreased IKAP/hELP1 expression persisted in daunomycin-treated cells for up to $4 \mathrm{~h}$ of treatment (Fig. 1B, top panel, compare lanes 3 and 4 and Fig. 2). Expression of hELP3, the catalytic subunit of the Elongator complex, was also decreased upon IKAP/hELP1 depletion (Fig. 1B, second panel from the top, compare lanes 3 and 4), in agreement with previous observations made in yeast [4] and in human cells [11]. Serine 15 of p53 is specifically phosphorylated by DNA-damaging drugs and this posttranslational modification stabilizes $\mathrm{p} 53$ by preventing its MDM2-mediated degradation [25]. Thus, we investigated to which extent this process was impaired upon IKAP/hELP1 depletion in HCT116 cells. As expected, the unphosphorylated and serine 15 phosphorylated forms of p53 levels were dramatically increased upon daunomycin treatment in GFP but also in IKAP/hELP1 shRNA cells (Fig. 1B, compare lanes 2 and 4 as well as lanes 1 and 3, second and third panels from the bottom). Therefore, these data indicate that Elongator deficiency does not significantly impair the DNA damagemediated stabilization of p53 through its phosphorylation on serine 15 in HCT116 cells.

p21 is a well known p53 target gene whose induced expression by some DNA-damaging agents triggers a cell cycle arrest $[26,27]$. Therefore, we first investigated whether IKAP/hELP1 is somehow involved in both basal or daunomycin-induced $p 21$ expression by comparing p21 mRNA or protein levels in IKAP/hELP1 shRNA versus GFP shRNA HCT116 cells. Whereas daunomycin indeed induced p21 expression both at the mRNA and at the protein levels (Fig. 2, top panels, compare lanes 2 and 5 with lanes 1 and 4), IKAP/hELP1 depletion did not impair both basal and signalinduced p21 expression, (Fig. 2, compare left and right panels, respectively), at least up to $4 \mathrm{~h}$ of daunomycin treatment. However, this result did not rule out the possibility that other candidates, yet to be identified, require Elongator for their basal or DNA-damage-induced expression.

To identify those candidates, we next subjected total RNA extracted from untreated or daunomycin-stimulated IKAP/ hELP1 shRNA and GFP shRNA HCT116 cells to micro-array analysis (data not shown). We hypothesized that the expression of some genes might be specifically deregulated upon Elongator deficiency. To identify those genes, micro-array analysis were also carried out using total RNA extracted from IKAP/hELP1 shRNA and GFP shRNA p53 deficient HCT116 cells as $\mathrm{p} 53$ plays a key role in the induction of several genes upon daunomycin treatment. IKAP/hELP1 protein levels remained unchanged upon daunomycin treatment in both wild type or p53 deficient cells (Fig. 3A, top panels, compare lanes 3 and 4 and lanes 7 and 8, respectively). Some genes such as tumor necrosis factor receptor superfamily, member 10D (TNFRSF10D), sestrin 2 (SESN2), and BTG family member 2 (BTG2) are known to be induced by a genotoxic stress through a p53-dependent pathway [28] and both our micro-array analysis and our real-time PCR experiments indeed confirmed that these genes were induced upon $2 \mathrm{~h}$ of daunomycin
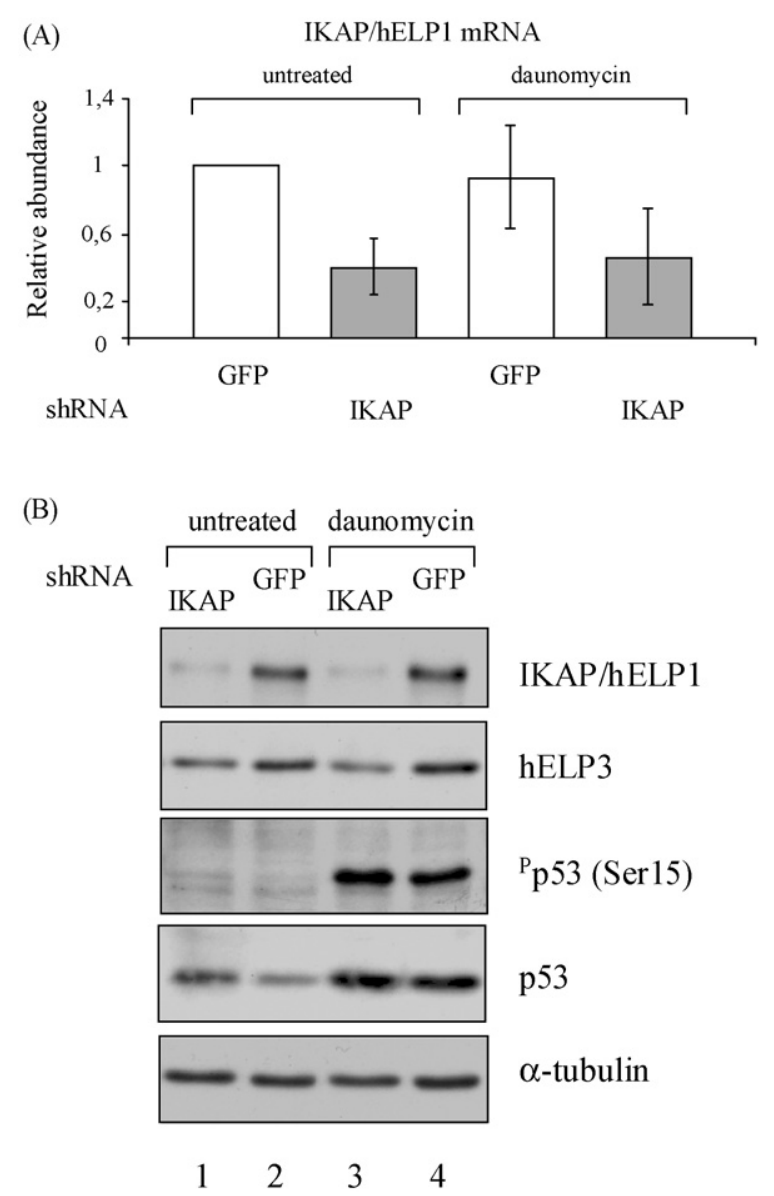

Fig. 1 - Elongator is dispensable for daunomycin-mediated p53 phosphorylation and subsequent stabilization in colon cancer-derived cells. (A and B) Stably infected IKAP/hELP1 and GFP shRNAs HCT116 cells were left untreated or stimulated with daunomycin $(1 \mu \mathrm{M})$ for $2 \mathrm{~h}$. (A) Residual IKAP/hELP1 mRNA was around $45 \%$ compared to control cells, as assessed by quantitative real-time PCR. The abundance of IKAP/hELP1 mRNA levels in unstimulated GFP shRNA HCT116 cells is set to 1 and the IKAP/hELP1 mRNA levels in the other experimental conditions are relative to that after normalization with beta-2-microglobulin (B2M) levels. Data from three independent experiments performed on three distinct infections are shown (mean values \pm S.D.). (B) Decreased IKAP/hELP1 expression triggers a modest $\mathrm{p} 53$ stabilization through serine 15 phosphorylation in both basal and daunomycin-stimulated cells. IKAP/hELP1 shRNA and GFP shRNA HCT116 cells were left untreated (lanes 1 and 2) or stimulated with daunomycin for $2 \mathrm{~h}$ (lanes 3 and 4) and cell extracts were subjected to anti-IKAP/hELP1, -ELP3, -serine 15 phospho p53 ( $\left.{ }^{\mathrm{p}} 553\right)$ and -p53 Western blot analysis, as indicated. An anti- $\alpha$-tubulin Western blot was performed as well for normalization purposes (bottom panel).

treatment in HCT116 cells (Fig. 3B, on the left). Of note, their levels of expression did not change upon IKAP/hELP1 depletion in both unstimulated or daunomycin-treated cells (Fig. 3B, on the left). On the other hand, mRNA levels of PKIB, a member 
(A) shRNA

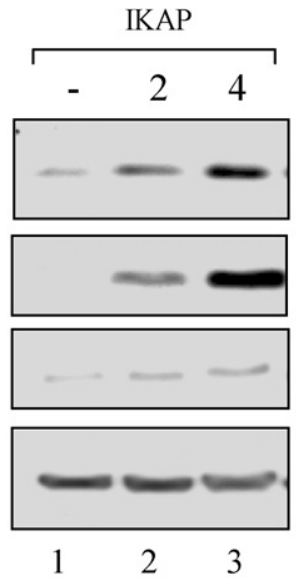

Daunomycin (h)

p21

p53

IKAP/hELP1

$\alpha$-tubulin
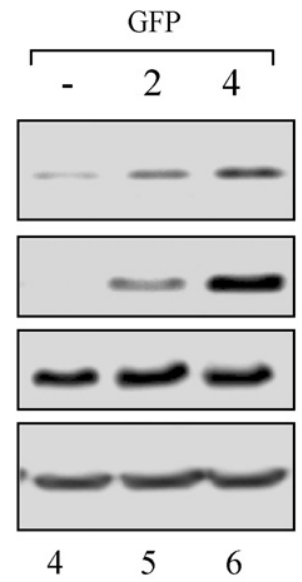

(B)

p21 mRNA

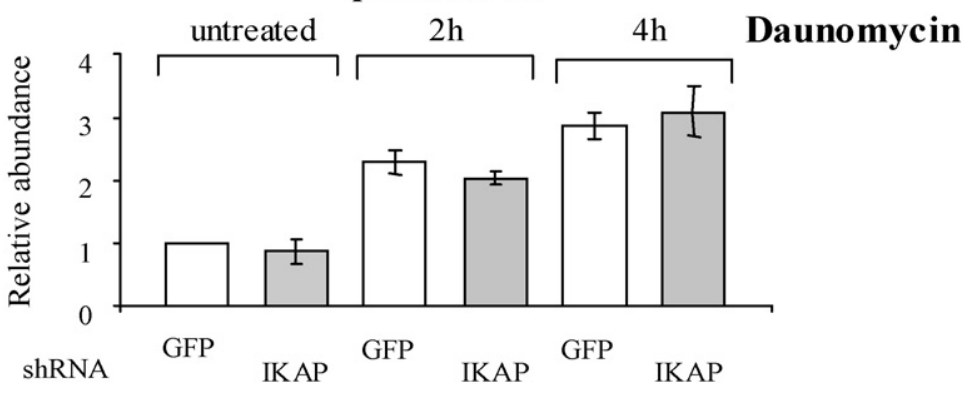

Fig. 2 - Elongator is dispensable for the early phase of daunomycin-induced p21 expression in HCT116 cells. Stably transduced IKAP/hELP1 shRNA (lanes 1-3) and GFP shRNA HCT116 cells (lanes 4-6) were left untreated or stimulated with daunomycin $(1 \mu \mathrm{M})$ for the indicated period of times. On the top, cell extracts were subjected to anti-p21, -p53, -IKAP/hELP1 and $-\alpha$-tubulin Western blot analysis. At the bottom, total RNAs were extracted from untreated or daunomycin-stimulated IKAP/hELP1 shRNA or GFP shRNA HCT116 cells as indicated and p21 mRNA levels were assessed by quantitative real-time PCR analysis. The abundance of p21 mRNA levels in unstimulated GFP shRNA HCT116 cells is set to 1 and the p21 mRNA levels in the other experimental conditions are relative to that after normalization with B2M levels. The figure shows the data from three independent experiments performed on two distinct infections (mean values \pm S.D.).

of the cAMP-dependent protein kinase inhibitor (PKI) family were downregulated upon hELP1/IKAP depletion in both unstimulated or daunomycin-treated cells (Fig. 3B, on the right). Interestingly, the decrease of gene expression upon IKAP/hELP1 depletion was cell-type-dependent as the genes identified in similar experiments performed in HeLa cells were not downregulated as in IKAP/hELP1 depleted HCT116 cells (data not shown). Even if we may expect Elongator to play identical roles in distinct cell types, the identity of the genes whose expression is Elongator-dependent differs from one cell type to another, as previously discussed [11].

We previously reported a list of gene candidates whose expression was enhanced upon IKAP/hELP1 depletion in HeLa cells [11], suggesting that Elongator may also inhibit gene expression through yet unclear but potentially indirect mechanisms. Thus, we next focused our attention on the candidates whose expression was induced upon IKAP/hELP1 depletion in HCT116 cells. Interestingly, a significant number of them included candidates known to be regulated by p53. Among them were BARD1, BAX, SGK, DUSP4 and NR2F2 (Fig. 3C). As expected these p53-dependent genes [29-31] were no longer induced upon IKAP/hELP1 depletion in p53 deficient HCT116 cells (Fig. 3C). Taken together, our data provide a link between Elongator deficiency and the induction of some but not all p53-dependent genes.

3.2. IKAP/hELP1 inhibits basal and daunomycin-induced SGK expression in HCT116 cells and in untransformed fibroblasts

Among the genes whose basal expression is induced upon IKAP/hELP1 depletion, we found SGK and this finding was indeed confirmed by real-time PCR analysis) (Fig. 4A). Because SGK expression is p53-inducible in some cell types [32], we next determined whether the enhanced SGK expression seen upon Elongator deficiency also occurred in p53 deficient HCT116 cells. Interestingly, SGK mRNA levels were not induced in daunomycin-treated HCT116 cells lacking p53 and IKAP/hELP1 depletion did not cause increased SGK expression in such cells (Fig. 4A). Therefore, our results suggest that enhanced SGK expression seen in Elongator deficient cells occurs through a p53-dependent pathway in colon cancer-derived cells.

We next explored whether a similar observation can be made in another cell type where p53 is functional and selected normal diploid human fibroblasts. Two distinct RNAi 
(A)

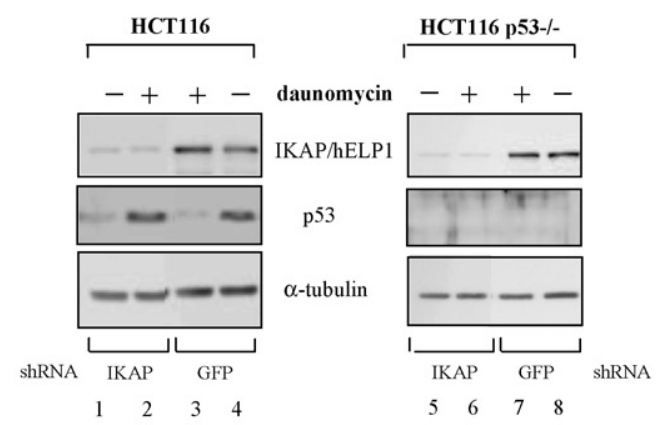

TNFRSF10D

(B)

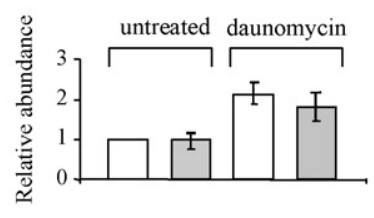

PKIB
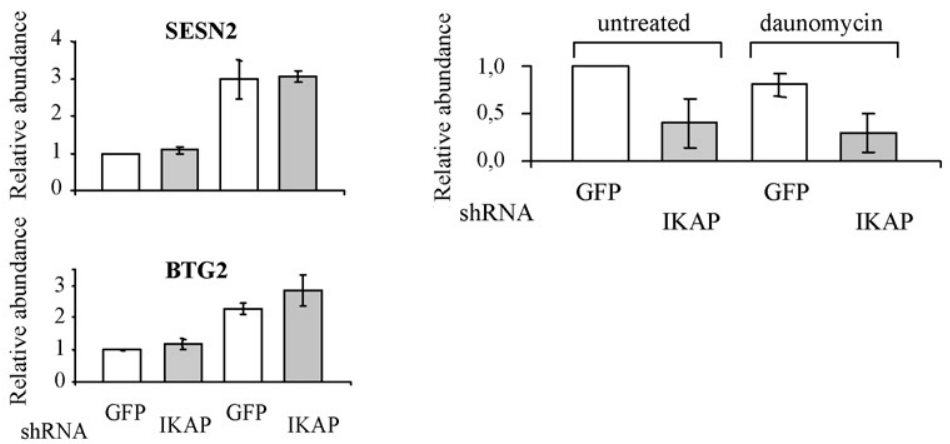

\begin{tabular}{|c|c|c|c|c|c|}
\hline (C) & \multirow[b]{2}{*}{ Accession number } & \multicolumn{2}{|c|}{$\begin{array}{l}\text { HCT116 p53 +/+ } \\
\text { IKAP versus GFP }\end{array}$} & \multicolumn{2}{|c|}{$\begin{array}{l}\text { HCT116 p53-/- } \\
\text { IKAP versus GFP }\end{array}$} \\
\hline Gene & & Fold induction & change p value & Foldinduction & change $p$ value \\
\hline BARD1 & NM_000465 & 3,2 & 0,000020 & 1 & 0,094279 \\
\hline BAX & NM_004324 & 2.8 & 0,000088 & 1 & 0,532344 \\
\hline SGK & NM_005627 & 2,5 & 0,000692 & 1 & 0,028766 \\
\hline DUSP4 & NM_001394 & 2,1 & 0,000020 & 1 & 0,500000 \\
\hline MSX1 & NM_002448 & 2,1 & 0,000020 & 1,4 & 0,000020 \\
\hline NR2F2 & NM_021005 & 2 & 0,000088 & 0,6 & 0,99998 \\
\hline
\end{tabular}

Fig. 3 - Elongator deficiency differentially regulates the expression of basal and daunomycin-induced genes in HCT116 cells and triggers the upregulation of selected p53-dependent genes. (A) Establishment of IKAP/hELP1 depleted wild type or p53 deficient HCT116 cells. Stably transduced IKAP/hELP1 shRNA and GFP shRNA wild type (lanes 1-4) or p53 deficient isogenic HCT116 cells (lanes 5-8) were left untreated or stimulated with daunomycin (1 $\mu \mathrm{M})$ for $2 \mathrm{~h}$. Protein cell extracts were subjected to anti-IKAP/hELP1, -p53 and - $\alpha$-tubulin Western blot analysis. (B) IKAP/hELP1 depletion causes impaired PKIB expression but not basal and daunomycin-induced expression of several p53-inducible target genes. mRNA levels of TNFRSF10D, SESN2, BTG2 and PKIB were assessed and represented as described in Fig. 2, using total RNAs isolated from untreated or daunomycin-stimulated IKAP/hELP1 shRNA or GFP shRNA HCT116 cells as indicated. The figure shows the data from three independent experiments generated from three independent infections (mean values \pm S.D.). (C) IKAP/ hELP1 depletion causes enhanced expression of selected p53 target genes. Micro-array analysis was performed using total RNAs isolated from untreated stably transduced IKAP/hELP1 shRNA or GFP shRNA wild type or p53 deficient HCT116 cells as indicated. The p53-dependent genes whose expression is increased in IKAP vs. GFP shRNA wild type or p53 deficient HCT116 cells are listed (left and right columns, respectively). Values represent the fold inductions in IKAP vs. GFP cells. Change $P$ values are mentioned as well. 
(A)

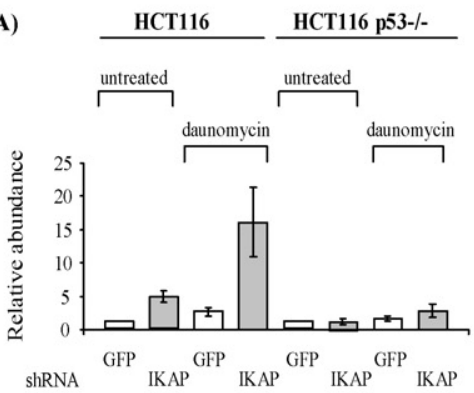

(C)

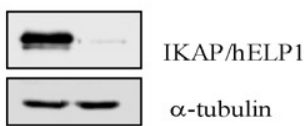

ShRNA GFP IKAP 2

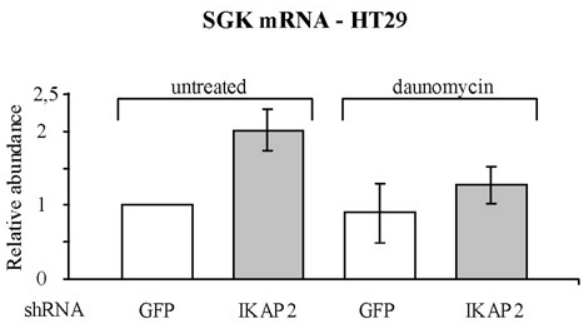

(B)

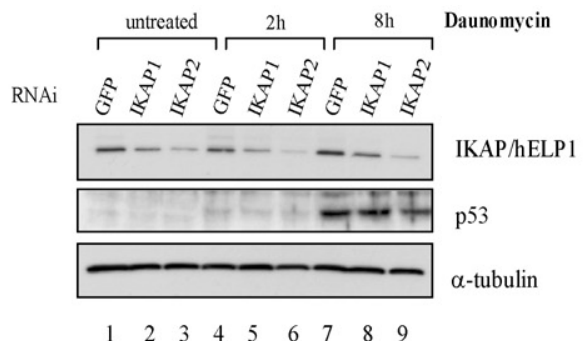

SGK mRNA - fibroblast

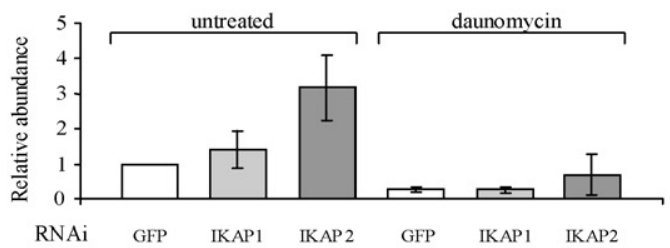

(D)

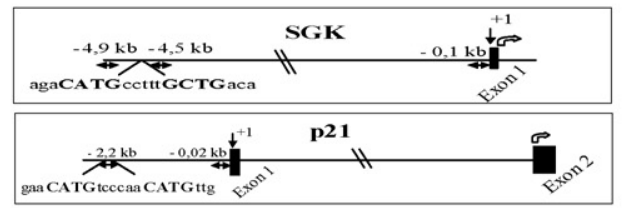

SGK promoter
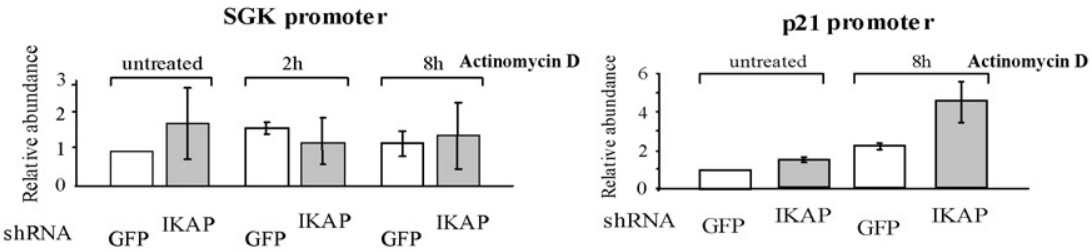

Fig. 4 - IKAP/hELP1 depletion enhances basal and daunomycin-induced SGK expression through a p53-dependent pathway. (A) Enhanced basal and daunomycin-induced SGK expression upon IKAP/hELP1 depletion in HCT116 cells. Stably transduced IKAP/hELP1 shRNA and GFP shRNA wild type or p53 deficient HCT116 cells were left untreated or stimulated with daunomycin $(1 \mu \mathrm{M})$ for $2 \mathrm{~h}$. Total RNAs from these cells were extracted and SGK mRNA levels were assessed by quantitative real-time PCR. The abundance of SGK mRNAs in unstimulated GFP shRNA cells was set to 1 and SGK mRNA levels in the other conditions were relative to that after normalization with B2M levels. The figure shows the data from three independent experiments generated from 3 (WT p53 cells) or 2 (p53 ${ }^{-1-}$ cells) independent lentiviral infections (mean values \pm S.D.). (B) Enhanced basal mRNA SGK expression in human primary fibroblasts depleted for IKAP/hELP1.

Untransformed human fibroblasts were transfected with a GFP RNAi or with two distinct RNAis targeting the IKAP/hELP1 transcript ("IKAP1" and "IKAP2") and left untreated or stimulated $48 \mathrm{~h}$ later with daunomycin (1 $\mu \mathrm{M})$ for the indicated periods of time. On the top, protein cell extracts were subjected to anti-IKAP/hELP1, -p53 and - $\alpha$-tubulin Western blot analysis. At the bottom, total RNAs from "GFP", "IKAP1" or "IKAP2" fibroblasts subsequently left untreated or stimulated with daunomycin $(1 \mu \mathrm{M})$ for $2 \mathrm{~h}$ were extracted and SGK mRNA levels were assessed by quantitative real-time PCR. The data are represented as described before. The figure shows the data from three independent experiments performed with three distinct RNAi transfections (mean values \pm S.D.). (C) Lack of dramatic increased SGK mRNA expression in IKAP/hELP1 depleted HT29 cells. Colon cancer-derived cells HT29 cells were infected with the pLL3.7 shRNA GFP or IKAP2 lentiviral construct and protein cell extracts were subjected to anti-IKAP/hELP1 and - $\alpha$-tubulin Western blot analyses to confirm IKAP/hELP1 depletion (on the left). On the right, pLL3.7 shRNA GFP or IKAP infected HT29 cells were left untreated or stimulated with daunomycin $(1 \mu \mathrm{M})$ for $2 \mathrm{~h}$ and SGK mRNA levels were assessed by quantitative real-time PCR using total RNAs extracted from these cells. The figure shows the data from two independent experiments performed on two distinct infections (mean values \pm S.D.). (D) RNA polymerase II recruitment on the TATA box of the SGK promoter is not deregulated upon IKAP/hELP1 deficiency. On the top, schematic representation of both the SGK and p21 genes. Exons are depicted by boxes whereas the transcription initiation site (+1) and the initial ATG (vertical arrows) are also indicated. The localization of the primers used for ChIP analysis is illustrated by horizontal arrows and their position are indicated in kilobase relative to the transcription initiation site. The potential p53 binding site on the SGK gene was identified using the GENOMATIXMatinspector program as was the previously identified p53 binding site on the $p 21$ gene. At the bottom, ChIP assays using an anti-RNA polymerase II antibody were performed with stably transduced IKAP/hELP1 shRNA and GFP shRNA HCT116 
sequences ("IKAP1" and "IKAP2") were used to target the IKAP/hELP1 transcript. As expected, p53 expression was induced upon stimulation with daunomycin (Fig. 4B, compare lanes 1-3 with lanes 7-9). Moreover and in agreement with results obtained in HCT116 cells, SGK mRNA levels were also enhanced upon IKAP/hELP1 depletion, especially in fibroblasts targeted with the "IKAP 2" RNAi sequence (Fig. 4B) which actually caused a more dramatic IKAP/hELP1 depletion compared to the "IKAP1" RNAi sequence (Fig. 4B, top panel, compare lane 1 with lanes 2 and 3). Of note, SGK expression was not induced upon daunomycin treatment (Fig. 4B) and this observation may reflect the inability of p53 to induce SGK expression in fibroblasts, as previously reported in the Rat-2 fibroblasts-derived cell line [32]. Finally, SGK expression was not daunomycin-inducible in colon cancer-derived HT29 cells where p53 is not functional and SGK expression was modestly enhanced in Elongator deficient cells despite robust IKAP/ hELP1 depletion (Fig. 4C). Thus, we identified SGK as a gene whose expression is enhanced upon Elongator deficiency in several cell types and this pathway requires wild type p53.

Because the transcriptional elongation of genes induced upon DNA-damaging agents involves elongation factors whose recruitment occurs in a gene and signal-specific manner, we next explored whether IKAP/hELP1 depletion impaired CDK9 and FACT levels of expression, as both of them are recruited to distinct regions of the $p 21$ locus in response to DNA damage [23]. IKAP/hELP1 depletion did not affect FACT and CDK9 levels of expression, both in untreated and daunomycin-stimulated HCT116 cells (Fig. S1, on the left, second and third panels from the top, compare lanes 1-3 with lanes 4-6). A similar conclusion was raised in p53 deficient HCT116 cells (Fig. S1, on the right). Moreover, preliminary data excluded a differential recruitment of CDK9 or FACT to the SGK locus upon Elongator deficiency, as evidenced by ChIP assays (data not shown). Therefore, the deregulated SGK expression seen in IKAP/hELP1 depleted HCT116 cells occurs in a CDK9 and FACT-independent pathway.

p53 induces gene transcription through binding to a welldefined consensus sequence [33]. Thus, we next investigated whether the p53-dependent SGK upregulation seen upon Elongator deficiency requires a direct binding of $\mathrm{p} 53$ to the SGK promoter. We took advantage of the GENOMATIX software (Matinspector) to identify potential p53-binding sequence within a $5000 \mathrm{bp}$ long region upstream of the transcriptional initiation site. One potential p53-binding site was identified on the SGK promoter, as was the previously characterized p53binding sequence found on the 21 promoter (Fig. 4D). Still, we did not see any p53 recruitment on the SGK promoter by ChIP analysis on this site whereas such $\mathrm{p} 53$ recruitment was indeed observed upon actinomycin D stimulation on the p21 promoter (data not shown). In order to see whether the SGK induction observed upon IKAP/hELP1 depletion occurs at the transcriptional level, we next investigated whether an enhanced recruitment of RNA polymerase II could underlie

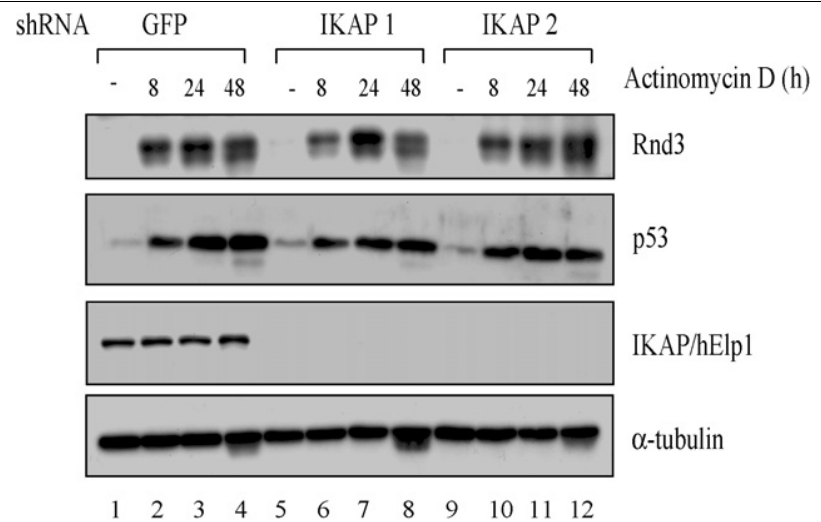

Fig. 5 - IKAP/hELP1 depletion does not alter actinomycin Dmediated RhoE/Rnd3 expression. HCT116 cells were infected with the pLL3.7 shRNA GFP, IKAP 1 [11] or the IKAP 2 lentiviral construct (which targets another sequence of the IKAP/hELP1 transcript) and the resulting infected cells were left untreated or stimulated with actinomycin $\mathrm{D}(20 \mathrm{nM})$ for the indicated periods of time. Anti-RhoE/Rnd3, -p53, -IKAP/hELP1 and - $\alpha$-tubulin Western blot analysis were carried out on the cell extracts.

the upregulated SGK expression seen in IKAP/hELP1 depleted cells. As expected, enhanced RNA polymerase II recruitment on the p21 promoter was observed in actinomycin Dstimulated shRNA GFP HCT116 cells, as previously described [23]. Of note, such recruitment was further enhanced in IKAP/ hELP1 shRNA HCT116 cells stimulated for $8 \mathrm{~h}$ with this DNAdamaging drug which means that p21 induction may be positively regulated upon Elongator deficiency in later times (Fig. 4D, right panel at the bottom). On the other hand, no significant changes for RNA polymerase II recruitment on the SGK promoter was observed upon Elongator deficiency (Fig. 4D, left panel at the bottom). Taken together, our results suggest that Elongator deficiency causes SGK upregulation through a transcriptional initiation-independent pathway.

Similarly to SGK, RhoE/Rnd3 is another p53-dependent and pro-survival gene induced upon DNA damage [34]. Because we systematically noticed that prolonged daunomycin treatments restored IKAP/hELP1 expression in IKAP/hELP1 shRNA cells (data not shown), we selected actinomycin $\mathrm{D}$ as another DNA-damaging agent. Therefore, we next explored whether RhoE/Rnd3 induction in actinomycin D-treated cells was somehow deregulated upon IKAP/hELP1 depletion. Whereas we indeed noticed that RhoE/Rnd3 protein expression levels were strongly induced upon actinomycin D stimulation in GFP shRNA HCT116 cells (Fig. 5, top panels, compare lanes 2-4 with lane 1), Elongator deficiency did not alter basal levels of RhoE/ Rnd3 (Fig. 5, top panel, compare lane 1 with lanes 5 and 9). Moreover, IKAP/hELP1 depletion did not interfere with the inducible RhoE/Rnd3 protein expression upon actinomycin D

cells which were left untreated or stimulated with actinomycin $\mathrm{D}(20 \mathrm{nM})$ for the indicated periods of time. Primers were derived from the nearest region of the transcription initiation site on both the SGK and $p 21$ genes, as explained above. The recruitment of RNA polymerase II in untreated GFP shRNA cells was set to 1 and its levels in the other conditions were relative to that after normalization with a non-coding region. The figure shows the data from three independent experiments performed with two distinct infections (mean values \pm S.D.). 
(A)
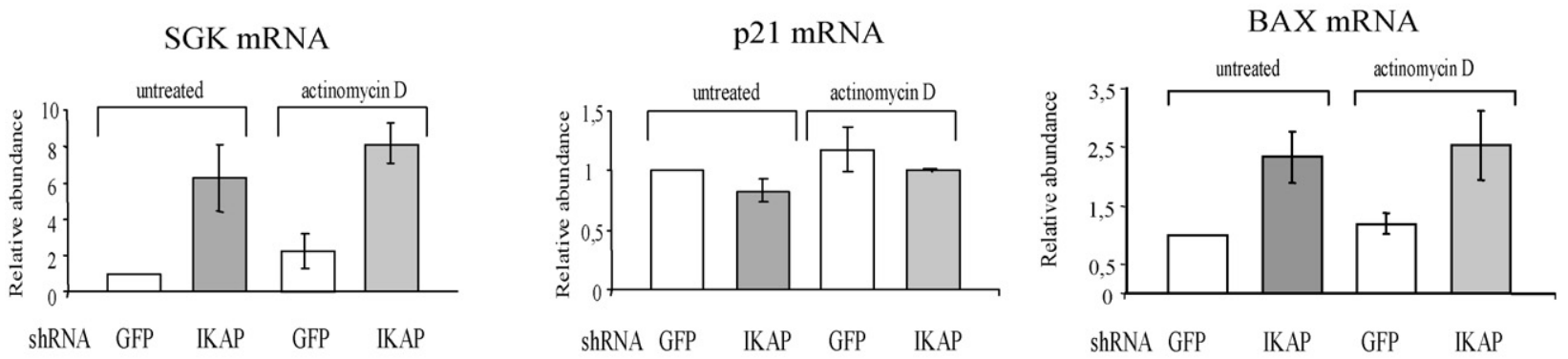

(B)

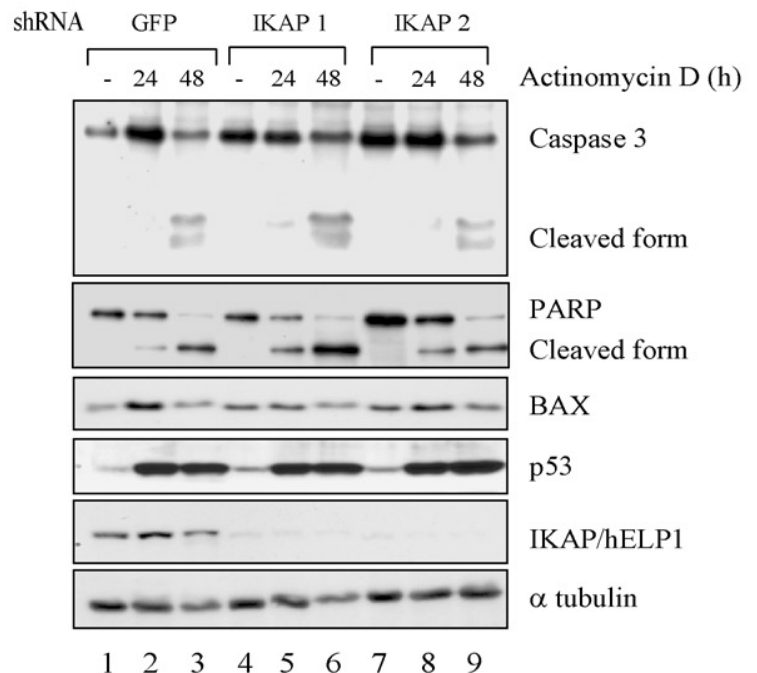

(C)

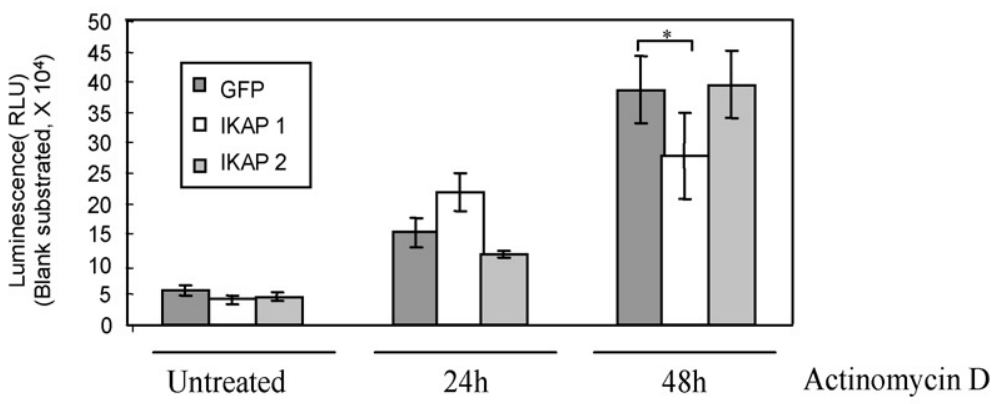

(D)

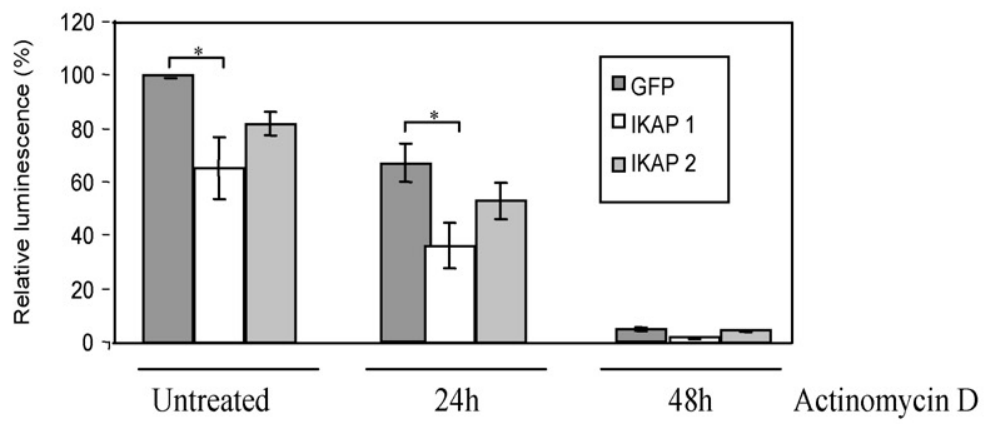

Fig. 6 - Elongator deficiency triggers enhanced BAX expression in HCT116 cells but does not modulate sensitivity to actinomycin D-mediated apoptosis. (A) IKAP/hELP1 depleted cells show enhanced expression of SGK and BAX but not p21 at the mRNA level. Stably transduced shRNA GFP or IKAP infected HCT116 cells were left untreated or stimulated with actinomycin $D(20 \mathrm{nM})$ for $2 \mathrm{~h}$ and the resulting total RNAs were subjected to quantitative real-time PCR in order to assess SGK, p21 and BAX mRNA levels, using the appropriate primers. The data are represented as described before. The figure 
stimulation (Fig. 5, top panel, compare lanes 6-8 and lanes 1012 with lanes $2-4$ ). Thus, this result suggests that some but not all pro-survival p53-dependent genes are regulated by Elongator.

3.3. Elongator deficiency does not significantly modulate the DNA damage-mediated apoptotic pathway in HCT116 cells

SGK is a pro-survival factor [35] but it was unclear whether its enhanced expression modulates the apoptotic response of IKAP/hELP1 depleted cells to DNA-damaging agent. Importantly and in contrast to $\mathrm{p} 21$, both mRNA and protein levels of BAX were enhanced upon IKAP/hELP1 depletion (Fig. 6A, middle and right panel, respectively and Fig. $6 \mathrm{~B}$, third panel from the top, compare lane 1 with lanes 4 and 7). Thus, two distinct p53 target genes coding for proteins acting as a survival (SGK) or as a pro-apoptotic product (BAX) have deregulated expression upon Elongator deficient HCT116 cells. To determine what the resulting outcome is in term of sensitivity to DNA damage-induced apoptosis, we assessed PARP and caspase 3 cleavages in IKAP/hELP1 shRNA versus GFP shRNA HCT116 cells upon stimulation with actinomycin D through Western blot analysis. In agreement with previous reports [36,37], actinomycin D triggered p53 stabilization, caspase 3 activation and PARP cleavage (Fig. 6B, fourth, first and second panel from the top, respectively, compare lane 1 with lanes 2 and 3). IKAP/hELP1 depletion did not significantly modulate caspase 3 activation nor PARP cleavage in response to actinomycin D (Fig. 6B, top and second panel from the top, compare lanes 2 and 3 with lanes 5 and 6 and with lanes 8 and 9). In agreement with those results, caspase 3 activities quantified by means of luminescent-based assays was indeed induced upon actinomycin $\mathrm{D}$ treatment but not affected by the IKAP/hELP1 depletion in HCT116 cells (Fig. 6C). Indeed, a modulation of caspase 3 activity upon $48 \mathrm{~h}$ of stimulation was only detected upon infection with one but not the other IKAP shRNA construct. As caspase 3 activation is intimately linked to cell apoptosis, we also noticed that IKAP/hELP1 depletion did not significantly modulate cell viability in actinomycin $\mathrm{D}$ versus untreated HCT116 cells (Fig. 6D). Taken together, our data demonstrate that Elongator deficiency triggers the activation of some but not all p53-dependent genes harbouring opposite functions with respect to apoptosis in colonderived cancer cells, which most likely explain why IKAP/ hELP1 depletion does not modulate the sensitivity of those cells to DNA-damaging agents.

\section{Discussion}

We demonstrate here that Elongator deficiency triggers the activation of selected p53-dependent genes in colon cancerderived cells, as evidenced by enhanced expression of BAX and SGK among other candidates. Moreover, we provide evidence that IKAP/hELP1 depletion does not significantly sensitize these cells to apoptosis triggered by a DNA-damaging agent. Therefore, we propose that the functional integrity of this complex is required to avoid inappropriate expression of some p53-dependent genes.

The molecular defects underlying impaired Elongator function only start to be elucidated. We show here that IKAP/hELP1 depletion have multiple consequences on the expression of several but not all p53-dependent genes. Indeed, IKAP/hELP1 depletion does not affect basal and DNA damageinduced $p 21$ and BTG2 expression levels. Because both proteins play critical roles for the p53-mediated cell cycle arrest [38], this observation suggests that Elongator is dispensable for this process. This conclusion is further supported by the fact that progression through the cell cycle does not appear to be altered upon Elongator deficiency (data not shown). Nevertheless, this issue deserves to be further investigated once totally deficient IKAP/hELP1 cells will be available, as we cannot rule out the possibility that residual IKAP/hELP1 levels seen in our experimental models may be sufficient to ensure proper progression through the cell cycle.

Elongator deficiency does not significantly affect the apoptotic status of the HCT116 cells subjected to a DNAdamaging agent but clearly triggers enhanced expression of selected p53-dependent genes. It is currently unclear whether this observation is exclusively due to impaired transcriptional elongation of selected target genes and/or to altered tRNA modifications and consequently to impaired translation of critical proteins. In any case, emerging data seem to indicate

shows the data from three independent experiments performed from two distinct lentiviral infections (mean values \pm S.D.). (B) Enhanced basal but not actinomycin D-induced BAX protein levels upon IKAP/hELP1 depletion in colon cancer-derived cells. HCT116 cells were infected with the pLL3.7 shRNA GFP, IKAP 1 [11] or the IKAP 2 lentiviral construct and the resulting infected cells were left untreated or stimulated with actinomycin $D(20 \mathrm{nM})$ for the indicated periods of time. Protein cell extracts were subjected to anti-IKAP/hELP1, -p53, -BAX, -caspase 3, -PARP and - $\alpha$-tubulin Western blot analysis. (C) Elongator deficiency does not modulate actinomycin D-induced cell apoptosis. HCT116 cells infected with the pLL3.7 shRNA GFP, IKAP 1 [11] or the IKAP 2 lentiviral construct were seeded in duplicates and left untreated or stimulated with actinomycin $\mathrm{D}(20 \mathrm{nM})$ for the indicated periods of time. Cellular apoptosis was quantified by assessing caspases $3 / 7$ activities. The figure shows the data obtained from three distinct lentiviral infections (mean values \pm S.D.). Statistical analysis of the data was performed by the one-way ANOVA with Dunn's multiple comparison test using the Prism 4.00 Software (Graph pad, San Diego, USA). P values $<0.05$ were considered as statistically significant. (D) IKAP/hELP1 depletion does not alter viability nor actinomycin D-induced cell death in colon cancer-derived cells. HCT116 cells infected with the pLL3.7 shRNA GFP, IKAP 1 [11] or the IKAP 2 lentiviral construct were seeded in duplicates and left untreated or stimulated with actinomycin $D(20 \mathrm{nM})$ for the indicated periods of time. Viable cells were counted and the resulting number was compared to the one obtained with the untreated HCT116 shRNA GFP cells as indicated in Section 2. The figure shows the data obtained from three distinct lentiviral infections (mean values \pm S.D.). 
that defects in both processes trigger a p53-activating stress response. Regarding transcriptional elongation, previous reports established a link between inhibition of transcription and induction of a p53-dependent cellular response $[37,39,40]$. Whereas these data were obtained using inhibitors of RNA synthesis such as actinomycin D [39], recent reports based on the generation of knock-out mice, undoubtedly provided further evidence for a link between impaired RNA synthesis and p53 activation. In this context, the in vivo invalidation of Elongin A, the transcriptionally active form of the elongation factor Elongin, is embryonic lethal because of senescence-like growth defects and massive apoptosis. Interestingly, the Elongin deficient embryos showed activation of the p53 signalling pathways [41]. The blockage of transcriptional elongation through micro-injection of antibodies that specifically recognized the elongating form of RNA polymerase II resulted in p53 accumulation in the nucleus, even in the absence of any DNA damage [40]. We did not observe a significant p53 accumulation upon Elongator deficiency in HCT116 cells but this is most likely due to the fact that only a limited number of genes are not properly transcribed upon Elongator deficiency [11]. Whether the enhanced p53-dependent gene expression seen upon IKAP/hELP1 depletion is due to slightly enhanced p53 levels seen in these cells is a likely explanation. Thus, our study, combined with these reports, collectively suggest that impaired transcriptional elongation triggers some p53-dependent pathways.

Because of accumulated evidence for a role of Elongator in tRNA modification [14], the activation of some p53-dependent genes seen upon IKAP/hELP1 depletion may also be due to impaired tRNA modifications and consequently to translational infidelity of yet to be identified proteins. In this context, an impaired ribosome function through conditional knock-out of the $40 \mathrm{~S}$ ribosomal protein S6 in T cells triggered defects in the fidelity of the translational machinery and consequently activated some p53-dependent checkpoint pathways to prevent aberrant cell division [42]. Which proteins would not be properly translated because of defects in tRNA modifications upon Elongator deficiency and how translational infidelity triggers selected $\mathrm{p} 53$ pathways remain unclear issues.

Elongator deficiency causes enhanced mRNA expression of candidates coding for proteins having opposite functions with respect to cell survival, such as BAX and SGK for example. This may explain why the enhanced BAX levels seen in IKAP/hELP1 depleted cells do not trigger more spontaneous apoptosis, despite the essential role played by this protein in the p53mediated pro-apoptotic pathway [43]. Of note however and in contrast to BAX, we failed to see enhanced SGK expression at the protein level upon IKAP/hELP1 depletion (data not shown). This may be due to the intrinsic instability of SGK, which is known to be quickly degraded through the proteasome pathway [44]. In any case, we clearly show here that the upregulated SGK mRNA expression requires wild type p53 as SGK is no longer induced in p53 deficient HCT116 or in HT29 cells, where p53 is not functional. SGK was previously identified as a p53-inducible kinase upon DNA damage [45]. Although a potential p53-responsive sequence was found in the SGK promoter, we have no evidence for $\mathrm{p} 53$ being recruited to this sequence in vivo, as judged by ChIP analysis. We actually do not support the hypothesis that enhanced SGK expression seen upon Elongator deficiency occurs at the transcriptional initiation step as the recruitment of the RNA polymerase II on the TATA box was not significantly modulated upon IKAP/hELP1 depletion in HCT116 cells. Thus, other mechanisms such as a more efficient transcriptional elongation or a stabilization of the SGK transcript may underlie SGK upregulation.

Although induced by the pro-apoptotic protein p53, SGK acts as a pro-survival factor through phosphorylation of FOXO3a [35]. Thus, this p53-dependent SGK induction can be seen as compensatory mechanism, as previously reported for other p53-dependent genes coding for pro-survival candidates such as COX-2, for example $[46,47]$. This negative feedback loop may also explain why the Elongator deficient cells are not significantly more sensitive to the DNA-damaging-mediated apoptotic pathway.

We previously reported that IKAP/hELP1 depletion in HeLa cells triggers the upregulation of some genes, many of which encoding proteins involved in amino acid metabolism [11]. Such conclusion was also raised based on micro-array analysis performed on strains with deletions of several subunits of the Elongator complex in S. cerevisiae [48]. Importantly, this later study also reported that Elongator deficiency in this experimental system also triggers the upregulation of a subset of genes known to be induced upon unfavorable, stressful conditions, such as growth in the presence of carcinogenic alkylating or oxidizing agents or when subjected to ionizing radiation [48]. As those agents and radiations are strong p53-inducers in mammalian cells, our data suggest that Elongator depletion ultimately induces the upregulation of stress-induced genes in yeast but also in mammalian cells, which might underlie a highly conserved molecular mechanism throughout evolution.

Elongator deficiency is linked to FD, a severe human neurodevelopmental and neurodegenerative genetic disorder [48]. The molecular defects underlying this disease only start to be uncovered and we previously suggested that cell motility defects of progenitor neural cells during embryogenesis may contribute to the observed phenotype of the FD patients [11]. The neurodegeneration seen in this disease most likely implies premature or enhanced cell death. To which extent p53 is involved in this process remains totally unknown. Our present report opens the possibility that the progression of FD to a neurodegenerative disease may also be caused by a deregulated activation of some p53-dependent genes.

\section{Acknowledgements}

The authors are grateful to Dr. C. Lambert for providing the normal diploid fibroblasts, to Dr. L. van Parijs for the gift of the pLL3.7 lentiviral construct, to Drs. J. Svejstrup and B. Vogelstein for the gift of the anti-ELP3 antibody and the p53 deficient HCT116 cells, respectively. AC and MPM are Research Associate and Senior Research Associate at the Belgian National Funds for Scientific Research (F.N.R.S.), respectively. IC and MG are "TELEVIE" Research Assistants whereas CC is a F.R.I.A. Research Assistant. This work was supported by grants from the F.N.R.S., TELEVIE, the Belgian Federation against Cancer, the Concerted Research Action Program (04/09-323, 
University of Liege), the Inter-University Attraction Pole 6/12 (Federal Ministery of Science), the "Centre Anti-Cancéreux" and the "Fonds Léon Frédéricq" (ULg).

\section{Appendix A. Supplementary data}

Supplementary data associated with this article can be found, in the online version, at doi:10.1016/j.bcp.2008.03.006.

\section{R E F E R E N C E S}

[1] Otero G, Fellows J, Li Y, de Bizemont T, Dirac AM, Gustafsson CM, et al. Elongator, a multisubunit component of a novel RNA polymerase II holoenzyme for transcriptional elongation. Mol Cell 1999;3:109-18.

[2] Hawkes NA, Otero G, Winkler GS, Marshall N, Dahmus ME, Krappmann D, et al. Purification and characterization of the human elongator complex. J Biol Chem 2002;277: 3047-52.

[3] Kim JH, Lane WS, Reinberg D. Human Elongator facilitates RNA polymerase II transcription through chromatin. Proc Natl Acad Sci USA 2002;99:1241-6.

[4] Petrakis TG, Wittschieben BO, Svejstrup JQ. Molecular architecture, structure-function relationship, and importance of the Elp3 subunit for the RNA binding of holoelongator. J Biol Chem 2004;279:32087-92.

[5] Wittschieben BO, Otero G, de Bizemont T, Fellows J, Erdjument-Bromage $\mathrm{H}$, Ohba $\mathrm{R}$, et al. A novel histone acetyltransferase is an integral subunit of elongating RNA polymerase II holoenzyme. Mol Cell 1999;4:123-8.

[6] Winkler GS, Kristjuhan A, Erdjument-Bromage H, Tempst P, Svejstrup JQ. Elongator is a histone $\mathrm{H} 3$ and $\mathrm{H} 4$ acetyltransferase important for normal histone acetylation levels in vivo. Proc Natl Acad Sci USA 2002;99:3517-22.

[7] Kristjuhan A, Walker J, Suka N, Grunstein M, Roberts D, Cairns BR, et al. Transcriptional inhibition of genes with severe histone h3 hypoacetylation in the coding region. Mol Cell 2002;10:925-33.

[8] Gilbert C, Kristjuhan A, Winkler GS, Svejstrup JQ. Elongator interactions with nascent mRNA revealed by RNA immunoprecipitation. Mol Cell 2004;14:457-64.

[9] Metivier R, Penot G, Hubner MR, Reid G, Brand H, Kos M, et al. Estrogen receptor-alpha directs ordered, cyclical, and combinatorial recruitment of cofactors on a natural target promoter. Cell 2003;115:751-63.

[10] Kouskouti A, Talianidis I. Histone modifications defining active genes persist after transcriptional and mitotic inactivation. EMBO J 2005;24:347-57.

[11] Close P, Hawkes N, Cornez I, Creppe C, Lambert CA, Rogister $\mathrm{B}$, et al. Transcription impairment and cell migration defects in elongator-depleted cells: implication for familial dysautonomia. Mol Cell 2006;22:521-31.

[12] Huang B, Johansson MJ, Bystrom AS. An early step in wobble uridine tRNA modification requires the Elongator complex. RNA 2005;11:424-36.

[13] Rahl PB, Chen CZ, Collins RN. Elp1p, the yeast homolog of the FD disease syndrome protein, negatively regulates exocytosis independently of transcriptional elongation. Mol Cell 2005;17:841-53.

[14] Esberg A, Huang B, Johansson MJ, Bystrom AS. Elevated levels of two tRNA species bypass the requirement for elongator complex in transcription and exocytosis. Mol Cell 2006;24:139-48.
[15] Svejstrup JQ. Elongator complex: how many roles does it play? Curr Opin Cell Biol 2007;19:331-6.

[16] Nelissen H, Fleury D, Bruno L, Robles P, De Veylder L, Traas $J$, et al. The elongata mutants identify a functional Elongator complex in plants with a role in cell proliferation during organ growth. Proc Natl Acad Sci USA 2005;102:7754-9.

[17] Slaugenhaupt SA, Gusella JF. Familial dysautonomia. Curr Opin Genet Dev 2002;12:307-11.

[18] Gold-von Simson G, Axelrod FB. Familial dysautonomia: update and recent advances. Curr Probl Pediatr Adolesc Health Care 2006;36:218-37.

[19] Anderson SL, Coli R, Daly IW, Kichula EA, Rork MJ, Volpi SA, et al. Familial dysautonomia is caused by mutations of the IKAP gene. Am J Hum Genet 2001;68:753-8.

[20] Slaugenhaupt SA, Blumenfeld A, Gill SP, Leyne M, Mull J, Cuajungco MP, et al. Tissue-specific expression of a splicing mutation in the IKBKAP gene causes familial dysautonomia. Am J Hum Genet 2001;68:598-605.

[21] Sims III RJ, Belotserkovskaya R, Reinberg D. Elongation by RNA polymerase II: the short and long of it. Genes Dev 2004;18:2437-68.

[22] Orphanides G, LeRoy G, Chang CH, Luse DS, Reinberg D. FACT, a factor that facilitates transcript elongation through nucleosomes. Cell 1998;92:105-16.

[23] Gomes NP, Bjerke G, Llorente B, Szostek SA, Emerson BM, Espinosa JM. Gene-specific requirement for P-TEFb activity and RNA polymerase II phosphorylation within the p53 transcriptional program. Genes Dev 2006;20:601-12.

[24] Olivier S, Close P, Castermans E, de Leval L, Tabruyn S, Chariot A, et al. Raloxifene-induced myeloma cell apoptosis: a study of nuclear factor-kappaB inhibition and gene expression signature. Mol Pharmacol 2006;69:1615-23.

[25] Shieh SY, Ikeda M, Taya Y, Prives C. DNA damage-induced phosphorylation of p53 alleviates inhibition by MDM2. Cell 1997;91:325-34.

[26] el-Deiry WS, Harper JW, O'Connor PM, Velculescu VE, Canman CE, Jackman J, et al. WAF1/CIP1 is induced in p53mediated G1 arrest and apoptosis. Cancer Res 1994;54: 1169-74.

[27] el-Deiry WS, Tokino T, Velculescu VE, Levy DB, Parsons R, Trent JM, et al. WAF1, a potential mediator of $\mathrm{p} 53$ tumor suppression. Cell 1993;75:817-25.

[28] Kannan K, Amariglio N, Rechavi G, Jakob-Hirsch J, Kela I, Kaminski N, et al. DNA microarrays identification of primary and secondary target genes regulated by $\mathrm{p} 53$. Oncogene 2001;20:2225-34.

[29] Irminger-Finger I, Leung WC, Li J, Dubois-Dauphin M, Harb J, Feki A, et al. Identification of BARD1 as mediator between proapoptotic stress and p53-dependent apoptosis. Mol Cell 2001;8:1255-66.

[30] Huang Q, Raya A, DeJesus P, Chao SH, Quon KC, Caldwell JS, et al. Identification of $\mathrm{p} 53$ regulators by genome-wide functional analysis. Proc Natl Acad Sci USA 2004;101: 3456-61.

[31] Shen WH, Wang J, Wu J, Zhurkin VB, Yin Y. Mitogenactivated protein kinase phosphatase 2: a novel transcription target of $\mathrm{p} 53$ in apoptosis. Cancer Res 2006;66:6033-9.

[32] Maiyar AC, Huang AJ, Phu PT, Cha HH, Firestone GL. p53 stimulates promoter activity of the sgk. serum/ glucocorticoid-inducible serine/threonine protein kinase gene in rodent mammary epithelial cells. J Biol Chem 1996;271:12414-22.

[33] Wei CL, Wu Q, Vega VB, Chiu KP, Ng P, Zhang T, et al. A global map of $\mathrm{p} 53$ transcription-factor binding sites in the human genome. Cell 2006;124:207-19.

[34] Ongusaha PP, Kim HG, Boswell SA, Ridley AJ, Der CJ, Dotto GP, et al. RhoE is a pro-survival p53 target gene that inhibits 
ROCK I-mediated apoptosis in response to genotoxic stress. Curr Biol 2006;16:2466-72.

[35] Brunet A, Park J, Tran H, Hu LS, Hemmings BA, Greenberg ME. Protein kinase SGK mediates survival signals by phosphorylating the forkhead transcription factor FKHRL1 (FOXO3a). Mol Cell Biol 2001;21:952-65.

[36] Chang D, Chen F, Zhang F, McKay BC, Ljungman M. Dosedependent effects of DNA-damaging agents on p53mediated cell cycle arrest. Cell Growth Differ 1999;10:155-62.

[37] Ljungman M, O'Hagan HM, Paulsen MT. Induction of ser15 and lys 382 modifications of $\mathrm{p} 53$ by blockage of transcription elongation. Oncogene 2001;20:5964-71.

[38] Rouault JP, Falette N, Guehenneux F, Guillot C, Rimokh R, Wang $Q$ et al. Identification of BTG2, an antiproliferative p53-dependent component of the DNA damage cellular response pathway. Nat Genet 1996;14:482-6.

[39] Ljungman M, Zhang F, Chen F, Rainbow AJ, McKay BC. Inhibition of RNA polymerase II as a trigger for the p53 response. Oncogene 1999;18:583-92.

[40] Derheimer FA, O'Hagan HM, Krueger HM, Hanasoge S, Paulsen MT, Ljungman M. RPA and ATR link transcriptional stress to p53. Proc Natl Acad Sci USA 2007;104:12778-83.

[41] Miyata K, Yasukawa T, Fukuda M, Takeuchi T, Yamazaki K, Sakumi K, et al. Induction of apoptosis and cellular senescence in mice lacking transcription elongation factor, Elongin A. Cell Death Differ 2007;14:716-26.
[42] Sulic S, Panic L, Barkic M, Mercep M, Uzelac M, Volarevic S. Inactivation of $\mathrm{S} 6$ ribosomal protein gene in T lymphocytes activates a p53-dependent checkpoint response. Genes Dev 2005;19:3070-82.

[43] Harris SL, Levine AJ. The p53 pathway: positive and negative feedback loops. Oncogene 2005;24: 2899-908.

[44] Bogusz AM, Brickley DR, Pew T, Conzen SD. A novel Nterminal hydrophobic motif mediates constitutive degradation of serum- and glucocorticoid-induced kinase-1 by the ubiquitin-proteasome pathway. FEBS J 2006;273:2913-28.

[45] You H, Jang Y, You-Ten AI, Okada H, Liepa J, Wakeham A, et al. p53-Dependent inhibition of FKHRL1 in response to DNA damage through protein kinase SGK1. Proc Natl Acad Sci USA 2004;101:14057-62.

[46] Han JA, Kim JI, Ongusaha PP, Hwang DH, Ballou LR, Mahale $\mathrm{A}$, et al. P53-mediated induction of Cox-2 counteracts p53or genotoxic stress-induced apoptosis. EMBO J 2002;21:5635-44.

[47] Benoit V, de Moraes E, Dar NA, Taranchon E, Bours V, Hautefeuille A, et al. Transcriptional activation of cyclooxygenase- 2 by tumor suppressor $\mathrm{p} 53$ requires nuclear factor-kappaB. Oncogene 2006;25:5708-18.

[48] Axelrod FB. Familial dysautonomia. Muscle Nerve 2004;29:352-63. 